\title{
UNIVERSALITY AND SCALING IN NETWORKS OF PERIOD-DOUBLING MAPS WITH A PACEMAKER
}

\author{
ANNA S. IVANOVA, SERGEY P. KUZNETSOV, AND ANDREW H. OSBALDESTIN
}

Received 10 October 2005; Accepted 8 January 2006

The networks of globally coupled maps with a pacemaker have been introduced. We consider a generalization of the Kaneko model with a pacemaker represented by a single period-doubling element coupled unidirectionally with a set of other mutually coupled cells. We also investigate the dynamics of a system of two unidirectionally coupled elements, which manifests a special type of critical behaviour, known as bicriticality, at the point of simultaneous transition to chaos in both subsystems. With the help of the renormalization group (RG), we show for a case of two mutually coupled bicritical maps with a pacemaker that there are two types of coupling: dissipative and inertial. We investigate the dynamics of a network with a pacemaker with two types of global coupling and the properties of universality and scaling in this system.

Copyright (c) 2006 Anna S. Ivanova et al. This is an open access article distributed under the Creative Commons Attribution License, which permits unrestricted use, distribution, and reproduction in any medium, provided the original work is properly cited.

\section{Introduction}

The study of networks composed of elements with different types of dynamics attracted much attention recently. In particular, after seminal works of Kaneko [5-7], a class of period-doubling maps with global coupling has been studied extensively. Such networks may serve as models for the description of dynamical phenomena in complex extended systems of different physical natures. For example, the peculiarities intrinsic to globally coupled maps have been observed, as reported, in electro-chemical experiments, in electronic, and in laser systems [3, 11, 12, 16, 19].

One of the main motivations for the study of globally coupled maps is the hope that it will aid the understanding of the operation of natural neural networks, such as human or animal brains. Then, the intrinsic nontrivial information processes could be not only understood, but also reproduced in technical devices. In this context it is worth noting the simplicity of the design of the global coupling, for example, in electronic and laser systems. 


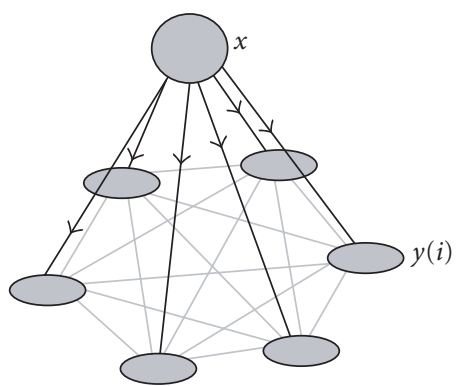

(a)

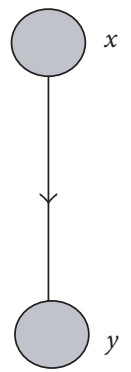

(b)

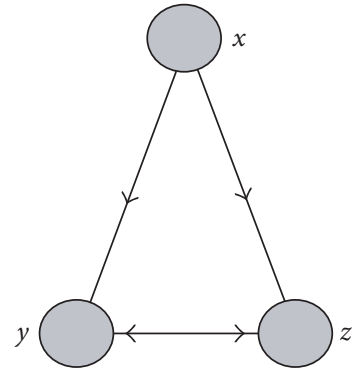

(c)

Figure 1.1. Schematic illustration of a network with global coupling, elements of which are under common pacemaker (a), two unidirectionally coupled maps (b), and two coupled maps under common pacemaker (c).

In natural excitable systems it is often the case that a particular element, or a part of the network of elements, may be identified as affecting and controlling the other elements. In biological studies it is called a pacemaker [18]. Apparently, it may be relevant in many cases for the functioning of natural excitable systems. Hence, it seems interesting to introduce such an additional element into the globally coupled map model of Kaneko and to reveal any novel dynamical properties. In this paper we consider a generalization of the Kaneko model with a pacemaker represented by a single period-doubling element coupled unidirectionally with a set of other mutually coupled cells. We note that the Feigenbaum quantitative universality intrinsic to period-doubling cascades in an isolated element implies universal scaling regularities in coupled systems and indeed in globally coupled networks. These matters have been previously considered using appropriate generalizations of Feigenbaum's renormalization group (RG) approach in $[1,2,9,13]$.

In Figure 1.1 the system is schematically shown. A pacemaker is represented as a logistic map, which is unidirectionally coupled with a set of globally coupled elements (Figure 1.1(a)). In Section 2, we first consider the dynamics of a system of two unidirectionally coupled elements (Figure 1.1(b)), which manifests a special type of critical behaviour, known as bicriticality, at the point of simultaneous transition to chaos in both subsystems. We then develop the renormalization group (RG) analysis for the case of a pacemaker driving two mutually coupled bicritical systems (Figure 1.1(c)). It will be shown that two types of coupling are relevant, dissipative and inertial, differing by their specific properties in terms of the RG transformation and scaling in the neighbourhood of the transition to chaos. In Section 3 we consider an arbitrary number of "slave" elements and investigate "clusterization" and dynamics near the bicritical situation.

\section{Renormalization group for systems with a pacemaker}

2.1. Dynamics of unidirectionally coupled maps. Let us start with the consideration of a system of two quadratic maps with unidirectional coupling:

$$
x_{n+1}=1-\lambda x_{n}^{2}, \quad y_{n+1}=1-A y_{n}^{2}-B x_{n}^{2} .
$$




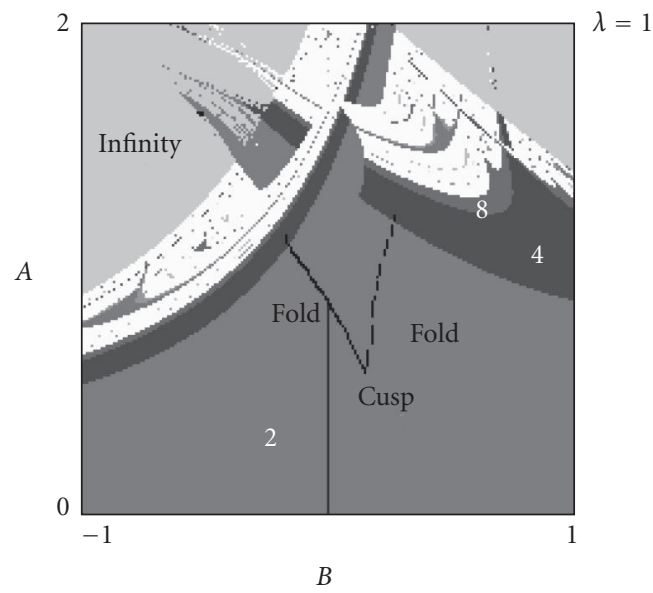

Figure 2.1. Parameter space $(A, B)$ of the slave system for fixed parameter of master system $\lambda=1$. Different tints mean domains of periodical behaviours, chaos is designated by white area. Numbers mean the period of oscillations. Firm and dotted lines are lines of folds (tangent bifurcations), which are crossed at cusp point.

Here the dynamical variables $x_{n}$ and $y_{n}$ relate to the master and the slave systems, respectively, $\lambda$ and $A$ are control parameters of the subsystems, and $B$ is the coupling coefficient. Dynamics of this model have been previously investigated in some respects in $[8,10,14]$.

First, note a property of multistability intrinsic to this system in some parameter domain. To explain this, let us assume for a moment that the coupling parameter $B$ is small enough, and that the values of $\lambda$ and $A$ are chosen in such a way so that each subsystem has a period-2 attractive orbit. Then, they may oscillate either in the same phase or in opposite phases depending on the initial conditions. Both states are stable and persist for nonzero coupling at least while it is not large enough. In Figure 2.1 a diagram of the $(A, B)$ parameter space is shown for fixed $\lambda=1$. At this $\lambda$, the master subsystem oscillates with period two, and it induces the same period in the slave system for very small $A$. With increase of $A$, a cascade of period-doubling bifurcations occurs followed by a transition to chaos. In the domain of period two there is a point of codimension 2 bifurcation, a cusp on the parameter plane $(A, B)$, at which two bifurcation lines meet, which correspond to tangent (saddle-node) bifurcations. In the domain between the folds bistability takes place.

If the parameter of the master system $\lambda$ is increased, the structure of domains in the parameter plane becomes more complex, but fundamental features of the picture persist. If in the master system chaotic behaviour arises, the slave system demonstrates forced chaotic oscillations.

In Figure 2.2 we show the $(\lambda, A)$ parameter space with constant coupling $B=0.375$. If parameter $\lambda$ of the master system is increased (corresponding to motion from left to right in the parameter plane), a cascade of period-doubling bifurcations in the master system 


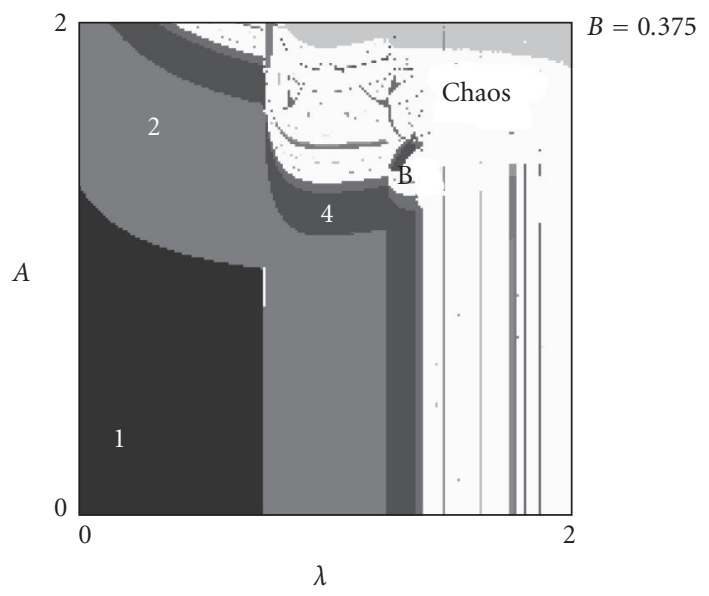

Figure 2.2. Parameter space $(\lambda, A)$ of the slave system for fixed parameter of coupling $B=0.375$. It is designated the bicritical point $B(1.401155,1.124981)$, which corresponds to simultaneous transition to chaos in both systems.

takes place. The bifurcation borders correspond to vertical lines in Figure 2.2. If we vary the parameter $A$ to move from bottom to top in the parameter plane, the dynamics of the master system remains unchanged, but in the slave system a cascade of period-doubling bifurcations takes place. If we increase both control parameters $A$ and $\lambda$, it is possible to get a situation of simultaneous transition to chaos in both subsystems. The point of transition to chaos in such case is called bicritical point [14]. It is designated by the letter $B$ in Figure 2.2. For coupling parameter $B=0.375$ the bicritical point has coordinates $\lambda=1.4011552, A=1.1249814$. At this point a special type of universal scaling behaviour occurs. The RG analysis of this situation has been performed in $[8,10,14]$.

The RG method consists in the following. Having the evolution operator of a system for a certain number of time steps, we can find a new evolution operator for doubled time interval and then rescale the dynamical variables to make the resultant operator as similar to the initial one as possible. This is just the RG transformation. We can repeat the process to obtain from the original operator a sequence of rescaled evolution operators for $2,4,8,16, \ldots$ units of discrete time. At the parameter values corresponding to the bicritical point the evolution operators asymptotically become invariant under the transformation. In other words, the operator sequence converges to a fixed point of the RG transformation. In the following discussion, dynamics at the critical point and in its vicinity obeys properties of universality and scaling. The universality arises because the fixed-point evolution operator is determined by the structure of the RG rather than by the actual form of an initial evolution operator. Scaling properties in parameter space are determined by spectrum of eigenvalues of the RG transformation linearized at the fixed point. Each essential eigenvalue with modulus exceeding unity is responsible for one essential parameter of system and gives a scaling factor for some direction of parameter space [14]. 
For the unidirectionally coupled maps the functional equations for the fixed point of the RG operator reads (see some details of derivation in the next section):

$$
g(x)=a g\left(g\left(\frac{x}{a}\right)\right), \quad f(x, y)=b f\left(g\left(\frac{x}{a}\right), f\left(\frac{x}{a}, \frac{y}{b}\right)\right) .
$$

Scaling properties of orbits in phase space in directions $x$ and $y$ are characterized by constants $a=-2.502907 \ldots$ and $b=-1.505318 \ldots$, respectively. In parameter space, the structure of domains in a small neighbourhood of the bicritical point manifests (asymptotically in small scales) self-similarity with respect to scale change along the horizontal and vertical axes in Figure 2.2 by the factors $\delta_{1}=-4.669201 \ldots$ and $\delta_{2}=2.392724 \ldots$.

\subsection{RG analysis of a system consisting of a master map and two coupled slave maps} near the bicritical point. Let us consider now a system consisting of one master map, the pacemaker, and two coupled slave maps (Figure 1.1(c)), and assume that the dynamical equations are of the following form:

$$
\begin{gathered}
x_{n+1}=g_{0}\left(x_{n}\right), \\
y_{n+1}=f_{0}\left(x_{n}, y_{n}\right)+\varepsilon \varphi\left(x_{n}, y_{n}, z_{n}\right), \\
z_{n+1}=f_{0}\left(x_{n}, z_{n}\right)+\varepsilon \varphi\left(x_{n}, z_{n}, y_{n}\right) .
\end{gathered}
$$

Here $\varphi(x, y, z)$ is a function of coupling which we require to vanish if the second and the third arguments coincide: $\left.\varphi(x, y, z)\right|_{z=y} \equiv 0$. The functions $g_{0}(x)$ and $f_{0}(x, y)$ are assumed to correspond to period-doubling mappings, for example, those presented in (2.2): $g_{0}(x)=1-\lambda x_{n}^{2}$ and $f_{0}(x, y)=1-A y_{n}^{2}-B x_{n}^{2}$, and the parameters are selected in such a way that without coupling both slave systems are precisely at the bicritical point.

Now we wish to apply RG approach to a situation similar to that developed earlier for coupled Feigenbaum period-doubling maps $[2,9,13]$.

As a preliminary step, we restrict the set of dynamical states under consideration to those close to symmetric states of both slave subsystems and assume that $\left|y_{n}-z_{n}\right| \ll 1$. We rewrite the equations using new variables:

$$
u=\frac{y+z}{2}, \quad v=\frac{y-z}{2}, \quad|v| \ll 1 .
$$

Then, we have

$$
\begin{gathered}
x_{n+1}=g_{0}\left(x_{n}\right), \\
u_{n+1}=f_{0}\left(x_{n}, u_{n}\right), \\
v_{n+1}=f_{0}^{\prime}\left(x_{n}, u_{n}\right) v_{n}+\varepsilon \phi_{0}\left(x_{n} ; u_{n}\right) v_{n} .
\end{gathered}
$$

Here $f_{0}^{\prime}(x, u)$ is the derivative of the function with respect to the second argument, and the coupling is characterized by the function

$$
\phi(x, y)=\lim _{y \rightarrow x} \frac{\varphi(x ; y, z)}{z-y} .
$$


6 Universality and scaling in networks

After two iterations, the set of variables $\{x, u, v\}$ obeys the following relations valid in the first order in $\varepsilon$ :

$$
\begin{aligned}
x_{n+2}= & g_{0}\left(g_{0}\left(x_{n}\right)\right), \\
u_{n+2}= & f_{0}\left(g_{0}\left(x_{n}\right), f_{0}\left(x_{n}, u_{n}\right)\right), \\
v_{n+2}= & f_{0}^{\prime}\left(g_{0}\left(x_{n}\right), f_{0}\left(x_{n}, u_{n}\right)\right) f_{0}^{\prime}\left(x_{n}, u_{n}\right) v_{n} \\
& +\varepsilon v_{n}\left[f_{0}^{\prime}\left(g\left(x_{n}\right), f_{0}\left(x_{n}, u_{n}\right)\right) \phi_{0}\left(x_{n}, u_{n}\right)+f_{0}^{\prime}\left(x_{n}, y_{n}\right) \phi_{0}\left(g_{0}\left(x_{n}\right), f_{0}\left(x_{n}, u_{n}\right)\right)\right] .
\end{aligned}
$$

Let us perform a scale change for the dynamical variables $x \rightarrow x / a, u \rightarrow u / b, v \rightarrow v / b$, where $a$ and $b$ are the universal factors mentioned in the previous section. This yields

$$
\begin{aligned}
x_{n+2}= & a g_{0}\left(g_{0}\left(a^{-1} x_{n}\right)\right), \\
u_{n+2}= & b f_{0}\left(g_{0}\left(a^{-1} x_{n}\right), f_{0}\left(a^{-1} x_{n}, b^{-1} u_{n}\right)\right), \\
v_{n+2}= & f_{0}^{\prime}\left(a^{-1} x_{n}, b^{-1} u_{n}\right) f_{0}^{\prime}\left(g\left(a^{-1} x_{n}\right), f_{0}\left(a^{-1} x_{n}, b^{-1} u_{n}\right)\right) v_{n} \\
& +\varepsilon v_{n}\left[f_{0}^{\prime}\left(g\left(a^{-1} x_{n}\right), f_{0}\left(a^{-1} x_{n}, b^{-1} u_{n}\right)\right) \phi_{0}\left(a^{-1} x_{n}, b^{-1} u_{n}\right)\right. \\
& \left.\quad+f_{0}^{\prime}\left(a^{-1} x_{n}, b^{-1} u_{n}\right) \phi_{0}\left(g_{0}\left(a^{-1} x_{n}\right), f_{0}\left(a^{-1} x_{n}, b^{-1} u_{n}\right)\right)\right] .
\end{aligned}
$$

In terms of new functions,

$$
\begin{aligned}
g_{1}(x)= & a g_{0}\left(g_{0}\left(a^{-1} x\right)\right), \\
f_{1}(x)= & b f_{0}\left(g_{0}\left(a^{-1} x\right), f_{0}\left(a^{-1} x, b^{-1} u\right)\right), \\
\phi_{1}(x, u)= & f_{0}^{\prime}\left(g\left(a^{-1} x\right), f_{0}\left(a^{-1} x, b^{-1} u\right)\right) \phi_{0}\left(a^{-1} x, b^{-1} u\right) \\
& +f_{0}^{\prime}\left(a^{-1} x, b^{-1} u\right) \phi_{0}\left(g_{0}\left(a^{-1} x\right), f_{0}\left(a^{-1} x, b^{-1} u\right)\right) .
\end{aligned}
$$

Equations (2.8) accept exactly the same form as (2.5):

$$
\begin{gathered}
x_{n+2}=g_{1}\left(x_{n}\right), \\
u_{n+2}=f_{1}\left(x_{n}, u_{n}\right), \\
v_{n+2}=f_{1}^{\prime}\left(x_{n}, u_{n}\right) v_{n}+\varepsilon \phi_{1}\left(x_{n} ; u_{n}\right) v_{n} .
\end{gathered}
$$

The relations (2.9) express the new functions in terms of the old ones and represent the RG transformation for the problem we consider. It corresponds to a passage from initial evolution operator for one iteration step to a renormalized operator for two steps. The procedure may be repeated again and again to get recursively the evolution operators for $4,8, \ldots$ steps of discrete time.

As the parameters in the functions $g_{0}(x)$ and $f_{0}(x, y)$ correspond to the bicritical point, the sequence of functional pairs $\left\{g_{k}(x), f_{k}(x, y)\right\}$ tends to the fixed point of the set of the 
functional equations

$$
g(x)=a g\left(g\left(a^{-1} x\right)\right), \quad f(x, u)=b f\left(g\left(a^{-1} x\right), f\left(a^{-1} x, b^{-1} u\right)\right) .
$$

The first is the well-known Feigenbaum-Cvitanović equation, and the second is equation derived and studied in $[8,10,14]$. High precision numerical solutions for these equations are known and published in the form of finite polynomial expansions [14].

In the remaining equation for the function of coupling, to study asymptotical behaviour of $\phi_{n}$, we have to substitute the functions $\{g(x), f(x, y)\}$ corresponding to the fixed point of the RG transformation with $\left\{g_{k}(x), f_{k}(x, y)\right\}$. The result is a functional equation with coefficients independent of $k$ :

$$
\begin{aligned}
v \phi_{n+1}(x, u)= & f^{\prime}\left(g\left(a^{-1} x\right), \tilde{f}\left(a^{-1} x, b^{-1} u\right)\right) \phi_{n}\left(a^{-1} x, b^{-1} u\right) \\
& +f^{\prime}\left(a^{-1} x, b^{-1} u\right) \phi_{n}\left(g\left(a^{-1} x\right), \tilde{f}\left(a^{-1} x, b^{-1} u\right)\right)
\end{aligned}
$$

The asymptotic behaviour will be determined by eigenfunctions for the eigenvalue problem obtained with a substitution $\phi_{n}(x, u) \mapsto \nu^{n} \phi(x, u)$ :

$$
\begin{aligned}
\nu \phi(x, u)= & f^{\prime}\left(g\left(a^{-1} x\right), \tilde{f}\left(a^{-1} x, b^{-1} u\right)\right) \phi\left(a^{-1} x, b^{-1} u\right) \\
& +f^{\prime}\left(a^{-1} x, b^{-1} u\right) \phi\left(g\left(a^{-1} x\right), \tilde{f}\left(a^{-1} x, b^{-1} u\right)\right) .
\end{aligned}
$$

We will show now that in a class of smooth functions $\phi(x, u)$ there are two relevant solutions which have eigenvalues larger than unity in modulus.

Let us search for a solution of (2.13) in a form $\phi(x, u)=f^{\prime}(x, u) H(x, u)$, where the prime sign designates the derivative with respect to the second argument. Using the expression $f^{\prime}(x, u)=f^{\prime}\left(a^{-1} x, b^{-1} u\right) f^{\prime}\left(g\left(a^{-1} x\right), f\left(a^{-1} x, b^{-1} u\right)\right)$ which follows from differentiating the second equation in (2.11) with respect to $u$, we obtain the following relation from (2.13):

$$
v H(x, u)=H\left(a^{-1} x, b^{-1} u\right)+H\left(g\left(a^{-1} x\right), \tilde{f}\left(a^{-1} x, b^{-1} u\right)\right) .
$$

Obviously, one eigenmode may be obtained as a trivial solution $H \equiv 1$ with eigenvalue $v_{1}=2$. One more relevant solution can be found from observation that the function $H(x, u)$ may have a singularity and behave as $H \propto u^{-1}$ near zero values of $u$. (Indeed, in this case the function $\phi(x, u)=f^{\prime}(x, u) H(x, u)$ remains smooth because $f^{\prime}(x, u) \propto u$. $)$ Substituting $H \propto u^{-1}$ into (2.14) we see that the singular terms must satisfy $v / u=b / u$, that is, $v_{2}=b=-1.50532 \ldots$. Then, in asymptotic of large $n$ the function $\phi_{n}(x, u)$ will behave as

$$
\phi_{n}(x, u)=C_{1} b^{n} \phi_{1}(x, u)+C_{2} 2^{n} \phi_{2}(x, u)
$$

where $C_{1}$ and $C_{2}$ are some coefficients depending on nature of coupling between two slave subsystems in the original model. Index 1 corresponds to the coupling we call inertial, and index 2 to that named dissipative. 


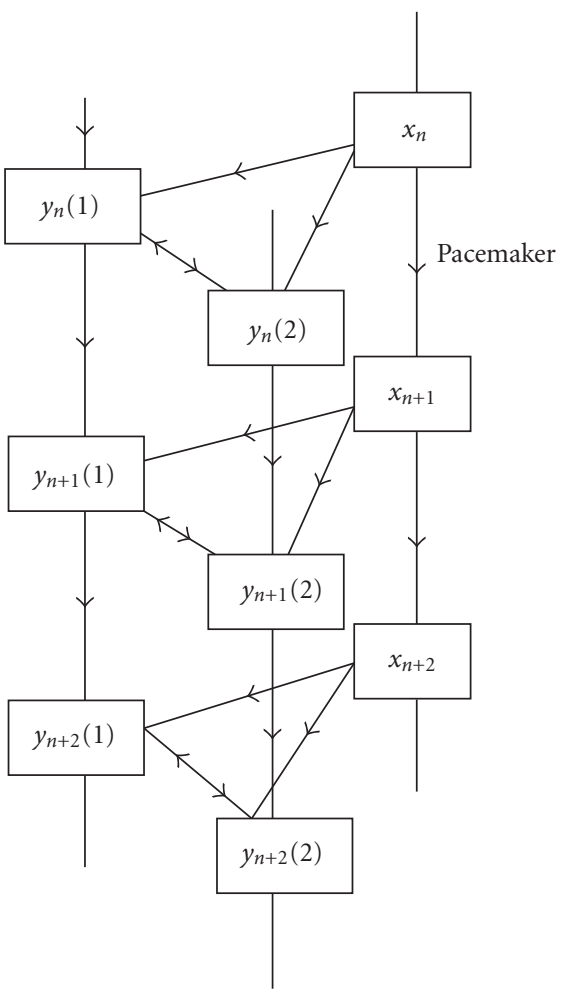

(a)

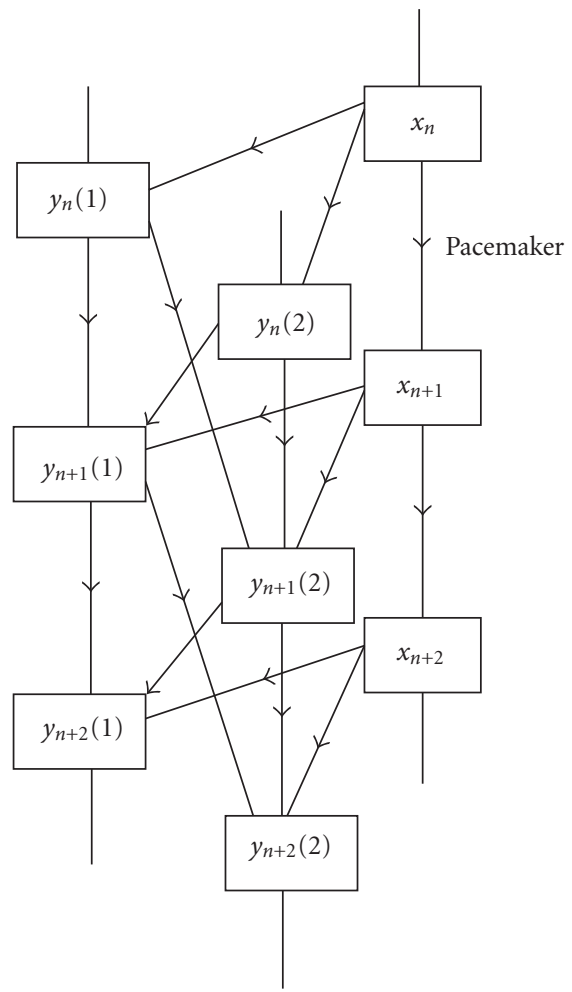

(b)

Figure 2.3. Schematic illustration of the two different types of coupling in system of two coupled maps with a pacemaker: dissipative (a) and inertial (b).

The meaning of two types of coupling is illustrated schematically in Figure 2.3. In the case of dissipative coupling, the instantaneous states of both subsystems mutually influence each other at a same step of discrete time. Such coupling tends to equalize the instantaneous states of both subsystems (Figure 2.3(a)). In the case of inertial coupling, one element influences another at a next time step. Such coupling keeps a memory about the state of the partner system on the previous step of evolution.

Let us consider the following model composed of a master and two coupled slave subsystems:

$$
\begin{gathered}
x_{n+1}=1-\lambda x_{n}^{2}, \\
y_{n+1}=1-A y_{n}^{2}-B x_{n}^{2}+\varepsilon_{i}\left(y_{n}-z_{n}\right)+A \varepsilon_{d}\left(z_{n}^{2}-y_{n}^{2}\right), \\
z_{n+1}=1-A z_{n}^{2}-B x_{n}^{2}+\varepsilon_{i}\left(z_{n}-y_{n}\right)+A \varepsilon_{d}\left(y_{n}^{2}-z_{n}^{2}\right) .
\end{gathered}
$$

Here the coupling between two slave subsystems is characterized by the coupling coefficients $\varepsilon_{i}$ and $\varepsilon_{d}$. It may be shown that the terms with $\varepsilon_{d}$ just correspond to dissipative 
coupling. The terms with $\varepsilon_{i}$ contain, in fact, a combination of inertial and dissipative coupling, with superior contribution of the first one. To arrange a situation of pure inertial coupling we must carefully choose the coefficients in (2.16) to exclude completely the dissipative coupling. (In other case, even in presence of very weak dissipative component, it will grow faster than the inertial component under subsequent application of the RG transformation and, hence, dominate the long-time dynamical behaviour of the system.)

In a concrete model, to estimate coefficients $C_{1}$ and $C_{2}$, that is, to analyse a "composition" of coupling, one can follow a method developed in [13] and consider multipliers (Floquet eigenvalues) for a set of unstable period $-2^{n}$ cycles dependent on $n$ in the limit of small coupling. Calculating eigenvalues of the derivative matrix for the mapping (6) at the fixed point of the equations $x_{*}=g\left(x_{*}\right), u_{*}=f\left(x_{*}, u_{*}\right)$, we obtain

$$
\begin{gathered}
\mu^{1}=g^{\prime}\left(x_{*}\right)=-1.60119 \ldots, \quad \mu^{2}=f^{\prime}\left(x_{*}, u_{*}\right)=-1.17885 \ldots, \\
\mu^{3}=f^{\prime}\left(x_{*}, u_{*}\right)+\varepsilon\left[C_{1} b^{n} D_{1}+C_{2} 2^{n} D_{2}\right],
\end{gathered}
$$

where $D_{i}=(1 / 2) \partial \phi_{i}(x, u) / \partial u$ at $x=x_{*}, u=u_{*}$.

It follows from (2.17) that, asymptotically, they must obey the equation $k=C_{1} b^{n} D_{1}+$ $C_{2} 2^{n} D_{2}$, and this allows to estimate the coefficients $C_{1}$ and $C_{2}$ via parameters of the model

$$
C_{1}=\varepsilon_{i}, \quad C_{2}=\varepsilon_{d}-0.11855 \varepsilon_{i}
$$

It is worth noting one important moment distinct for critical behaviour of the coupled systems with a pacemaker and those without it. At the Feigenbaum critical point in a system without pacemaker, the eigenvalue associated with the inertial coupling is larger in modulus than that for the dissipative coupling [13]. Therefore, in a case of weak coupling of general form (combination of the inertial and dissipative couplings) structure of the parameter space near the critical point is determined mainly by the inertial coupling which dominates in the long-time evolution operator. In contrast, in our case of two coupled slave subsystems at the bicritical point, the dissipative coupling is dominant and essential for the long-time behaviour in the case of weak coupling of general form.

In Figures 2.4(a) and 2.4(b) the $(\lambda, \varepsilon)$, we present parameter plane diagrams for the model with pure dissipative (Figure 2.4(a)) and pure inertial (Figure 2.4(b)) couplings. Different tones of grey represent periodic behaviour of different periods (indicated with numbers), and white corresponds to chaos. If the parameter $\lambda$ is increased, a cascade of period-doubling bifurcations occurs. In the pure dissipative case, the chaotic dynamics takes place at the $\lambda<\lambda_{c}$ by increasing the coupling parameter. In the pure inertial case, for small $\lambda$ the dynamics remain regular, but the bifurcation borders have more complicated form than in the pure dissipative coupling case.

In Figure 2.4 the illustrations of scaling in the $(\lambda, \varepsilon)$ parameter space for pure dissipative (left side) and pure inertial (right side) couplings are shown. As stated previously, the values of the other parameters correspond to the bicritical point $(A=1.1249$ and $B=$ $0.375)$. Each successive picture shows a magnification of the previous one. The following property of scaling holds. At the point of the parameter space $\left(\Delta \lambda / \delta_{1}, \Delta A / \delta_{2}, C_{1} a^{-1}\right.$, $\left.C_{2} 2^{-1}\right)$ dynamical regimes of the same kind as those found at the point $\left(\Delta \lambda, \Delta A, C_{1}, C_{2}\right)$ 


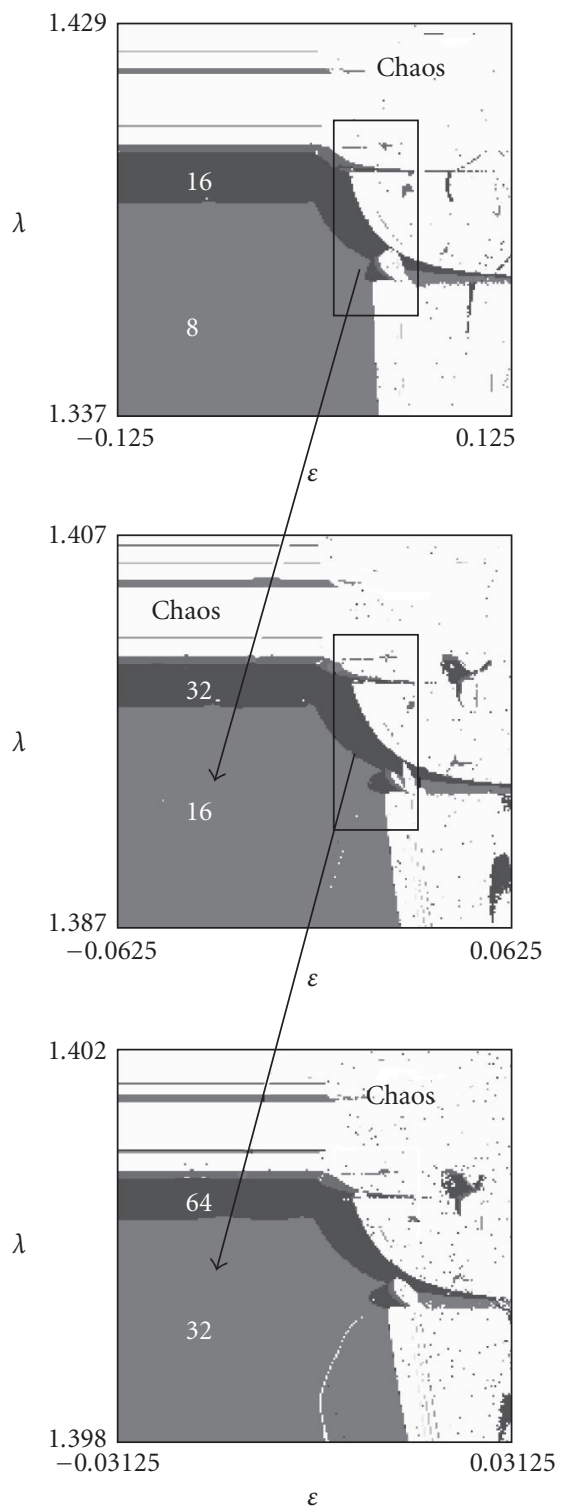

(a)

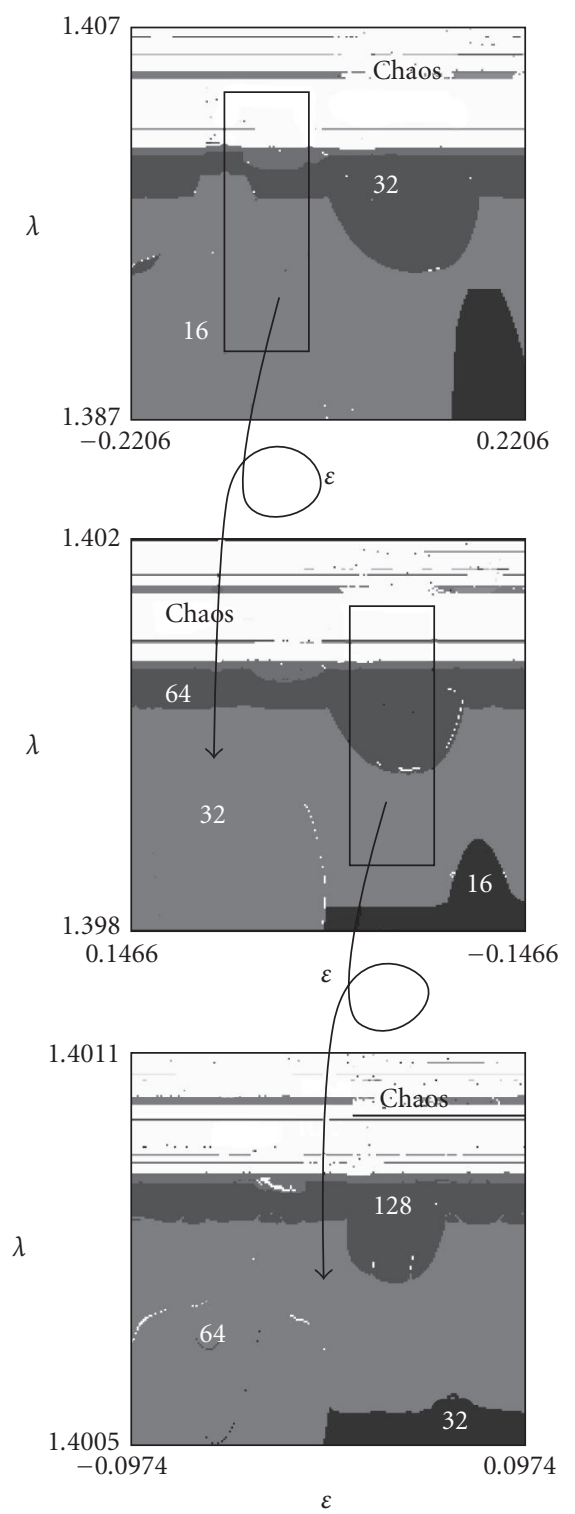

(b)

Figure 2.4. Illustrations of a scaling in the parameter space $(\lambda, \varepsilon)$ for only dissipative (a) and inertial (b) couplings for the following values of rest parameters $A=1.1249, B=0.375$, which correspond to the bicritical point. Different tints of grey colour mean periodic behaviour, white area-chaos. Numbers mean the different periods of oscillations. One can see that after special rescaling we have a similar structure near the bicritical point.

arise, but with doubled time scale, and initial conditions for dynamical variables obtained by rescaling with factors $a=-2.5029 \ldots$ for $x$ and $b=-1.5053 \ldots$ for $y$ and $z$. We can see 
that after rescaling by these factors, the structure of parameter space in neighbourhood of bicritical point is very close to the original.

\section{Globally coupled maps with a pacemaker}

3.1. Clusterization and equations for dynamics of clusterized states. Now, we may turn to a system of globally coupled maps with a pacemaker. As stated in the previous section, there are two relevant types of coupling between two slave subsystems, dissipative and inertial couplings. By imposing coupling by means of linear and quadratic terms for each pair of a large collection of the slave subsystems, we arrive to a model of a globally coupled network with a pacemaker:

$$
\begin{gathered}
x_{n+1}=1-\lambda x_{n}^{2} \\
y_{n+1}(i)=\left(1-\varepsilon_{d}\right) f\left(x_{n}, y_{n}(i)\right)-\varepsilon_{i} y_{n}(i)+\varepsilon_{i} N^{-1} \sum_{j=1}^{N} y_{n}(j)+\varepsilon_{d} N^{-1} \sum_{j=1}^{N} f\left(x_{n}, y_{n}(j)\right) .
\end{gathered}
$$

Last two terms in (3.1) are the same for all elements of the network and may be regarded as "mean fields" associated with two types of coupling

$$
F_{n}^{(1)}=N^{-1} \sum_{j=1}^{N} y_{n}(j), \quad F_{n}^{2}=N^{-1} \sum_{j=1}^{N} f\left(x_{n}, y_{n}(j)\right) .
$$

In accordance with the relation (2.18), we set

$$
\varepsilon_{i}=C_{1}, \quad \varepsilon_{d}=C_{2}+0.11855 C_{1},
$$

where $C_{1}$ corresponds to the pure inertial coupling and $C_{2}$ to the dissipative one.

As known, in the model without pacemaker a phenomenon takes place and it is called clusterization $[4,6]$. It consists in spontaneous formation of groups of cells, clusters, in such way that instantaneous states of elements relating to the same cluster coincide exactly. This is possible due to the global nature of coupling because neither the states of the elements, nor the effecting mean field differ for the elements from the same cluster. The same phenomenon obviously occurs in our model because a pacemaker equally influences all slave elements in the network. One can classify possible regimes of the system by a number of clusters $K$ and their relative population numbers. The relative population number of a cluster is defined as ratio of a number of cells in the cluster to the whole number of the cells in the network, $P_{m}=N_{m} / N$, where subscript $m$ specifies the cluster.

Following Kaneko, we distinguish in our system four types of clusterized regimes.

(1) Coherent attractor: all elements of the network relate to one cluster, that is, have identical instantaneous states.

(2) Attractors, which have a relatively small number of clusters, each containing a large number of elements comparable to the whole number of cells in the network.

(3) Attractors, which have both large clusters (population numbers of order 1) and small clusters (population numbers of order $N^{-1}$ ). 
(4) Attractors with a large number of clusters with population numbers of order $N^{-1}$.

Additionally, states of the network may be classified with respect to a type of dynamics in time. Of particular significance is the nature of the regime of the pacemaker. Remember that at small $\lambda$ the map $x_{n+1}=1-\lambda x_{n}^{2}$ has an attractive fixed point. In this case dynamics of the globally coupled slave maps is precisely analogous to that described by Kaneko in [6]. If $\lambda$ varies in an interval from 0.75 to 1.25 , regular dynamics of period 2 for all elements of globally coupled maps occurs, from 1.25 to 1.368 period 4 , takes place with period 4 and so on. On the basis of these regimes clusterized states of the network may be obtained with different periods and with chaotic dynamics of the slave subsystems by increasing the control parameter $A$. If $\lambda$ is larger than $\lambda_{c} \approx 1.401 \ldots$, the pacemaker demonstrates chaos (with exception of the so-called windows of regularity), and induces chaos-like dynamics of the excited elements. It may be synchronous chaos, which corresponds to type-1 regime (coherent state), or dynamics corresponding to types 2-4.

Let us suppose that we have a state of the network of the slave elements with $k$ clusters. In this case (3.1) may be rewritten as

$$
\begin{gathered}
x_{n+1}=1-\lambda x_{n}^{2} \\
Y_{n+1}^{k}=\left(1-\varepsilon_{2}\right) f\left(x_{n}, Y_{n}^{k}\right)-\varepsilon_{1} Y_{n}^{k}+\varepsilon_{1} \sum_{k=1}^{K} p_{k} Y_{n}^{k}+\varepsilon_{1} N^{-1} \sum_{j=1}^{N} f\left(x_{n}, Y_{n}^{k}\right),
\end{gathered}
$$

where $k=1,2, \ldots, K$, and a variable $Y_{n}^{k}$ relates to a cluster number $k$.

The simplest example is a two-cluster state. The equation (3.4) then takes the following form:

$$
\begin{gathered}
x_{n+1}=1-\lambda x_{n}^{2}, \\
Y_{n+1}^{k}=\left(1-\varepsilon_{2}\right) f\left(x_{n}, Y_{n}^{k}\right)+\varepsilon_{1} p_{2}\left(Y_{n}-Z_{n}\right)+\varepsilon_{1} p_{2}\left(f\left(x_{n}, Z_{n}\right)-f\left(x_{n}, Y_{n}\right)\right), \\
Z_{n+1}^{k}=\left(1-\varepsilon_{2}\right) f\left(x_{n}, Z_{n}^{k}\right)+\varepsilon_{1} p_{1}\left(Z_{n}-Y_{n}\right)+\varepsilon_{1} p_{1}\left(f\left(x_{n}, Y_{n}\right)-f\left(x_{n}, Z_{n}\right)\right),
\end{gathered}
$$

where relative population numbers are $P_{1}$ and $P_{2}=1-P_{1}$.

In the case $p_{1}=p_{2}=1 / 2$ the equations for two-cluster state become the same as for the case of two symmetric coupled maps with a pacemaker, the dynamics of which was described in previous section. In addition, now it is interesting to investigate how the asymmetry of population of two clusters influences the dynamics of the system.

In Figures 3.1 and 3.2 illustrations of scaling in different parameter space cross-sections are shown for pure dissipative (left side) and pure inertial (right side) couplings. If we compare Figure 2.4 (symmetric case) and Figure 3.1 (asymmetric case), for pure dissipative coupling, the domain of chaotic dynamics at $P=0.25$ becomes more narrow, that is, a nonsymmetric distribution of the elements in the clusters gives rise to more regular dynamics in the two-cluster state. For pure inertial coupling the borders of bifurcations in nonsymmetric case become more complicated.

In diagrams of the parameter plane $(\lambda, p)$ in Figure 3.2 for pure dissipative coupling, the structure of parameter space almost does not change with the variation of the population number; for pure inertial coupling the essential change of the structure occurs near the point of transition to chaos. 
Anna S. Ivanova et al.

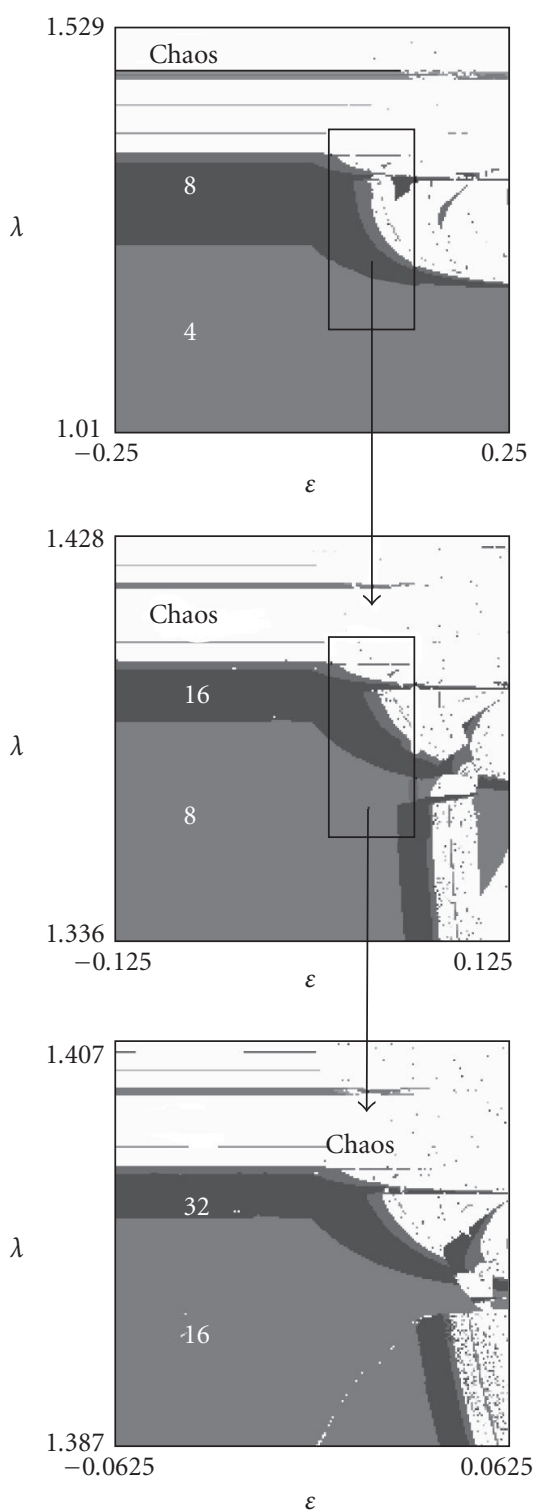

(a)

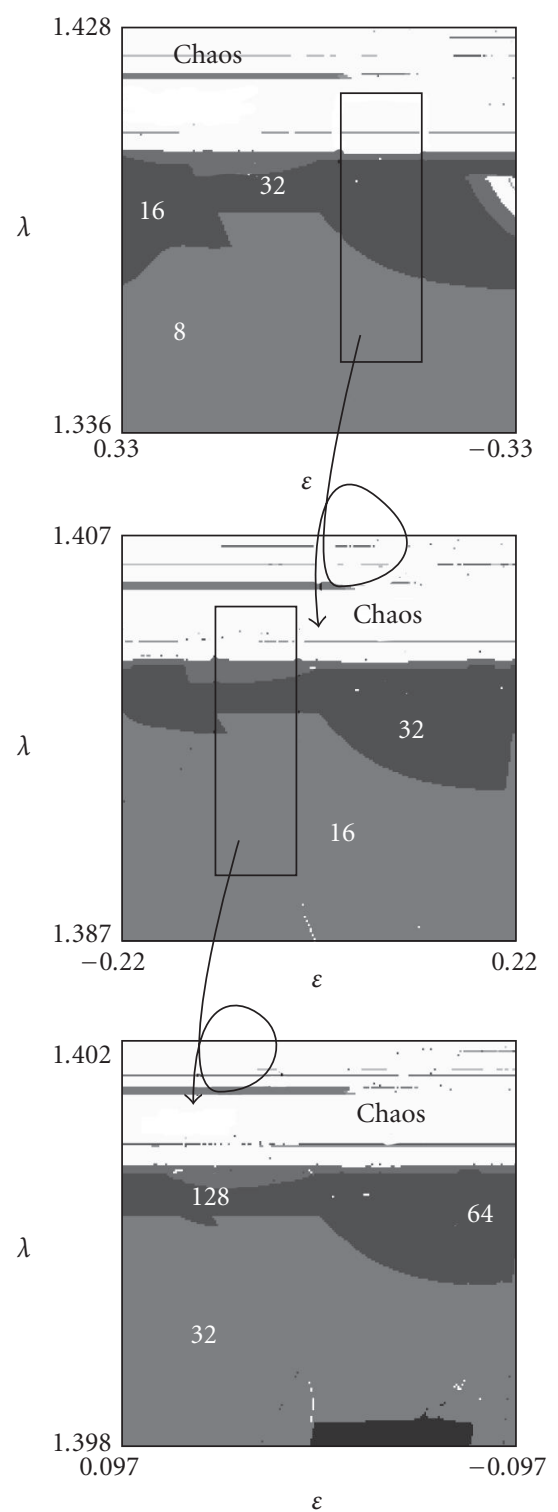

(b)

Figure 3.1. Illustrations of a scaling at the parameter space $(\lambda, \varepsilon)$ for pure dissipative (left side) and pure inertial (right side) couplings for the following values of the rest parameters $A=1.1249, B=$ 0.375 , which correspond to the bicritical point and $p_{1}=0.25$. Different tints of grey colour mean periodic behaviour, white area-chaos. Numbers mean the different periods of oscillations. One can see that after special rescaling we have a similar structure near the bicritical point.

Each next picture in Figures 3.1 and 3.2 represents an enlarged fragment of a previous diagram. Enlargement corresponds to factors of scaling obtained in the previous 


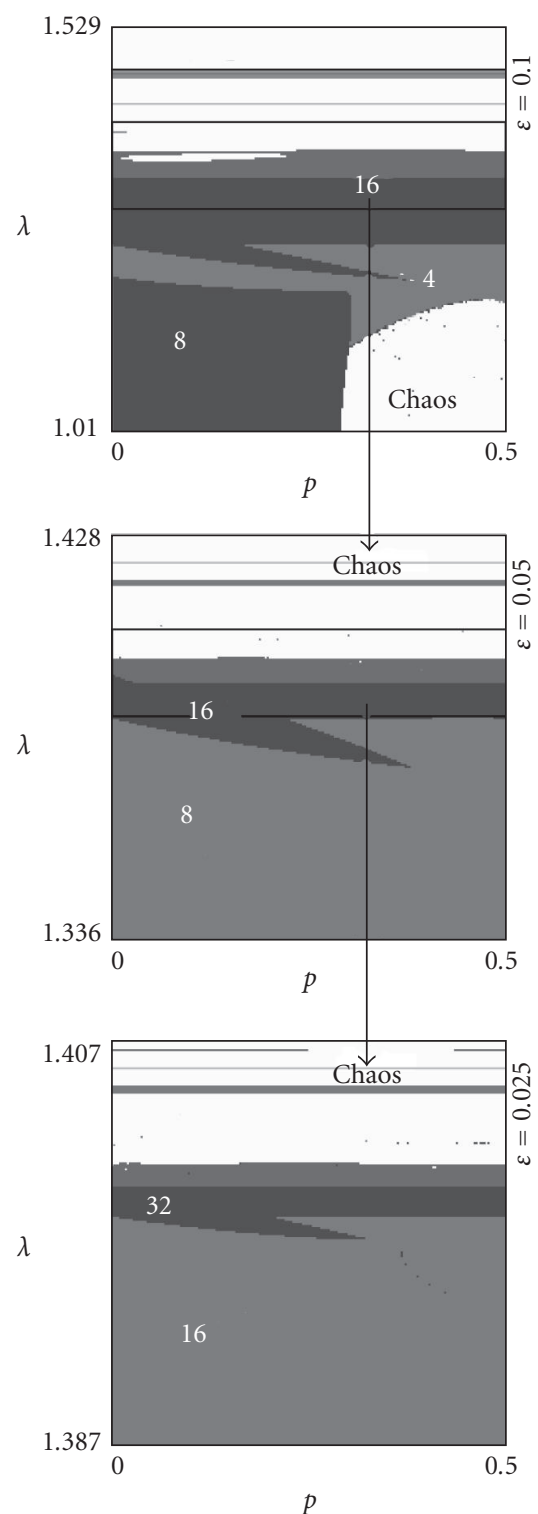

(a)

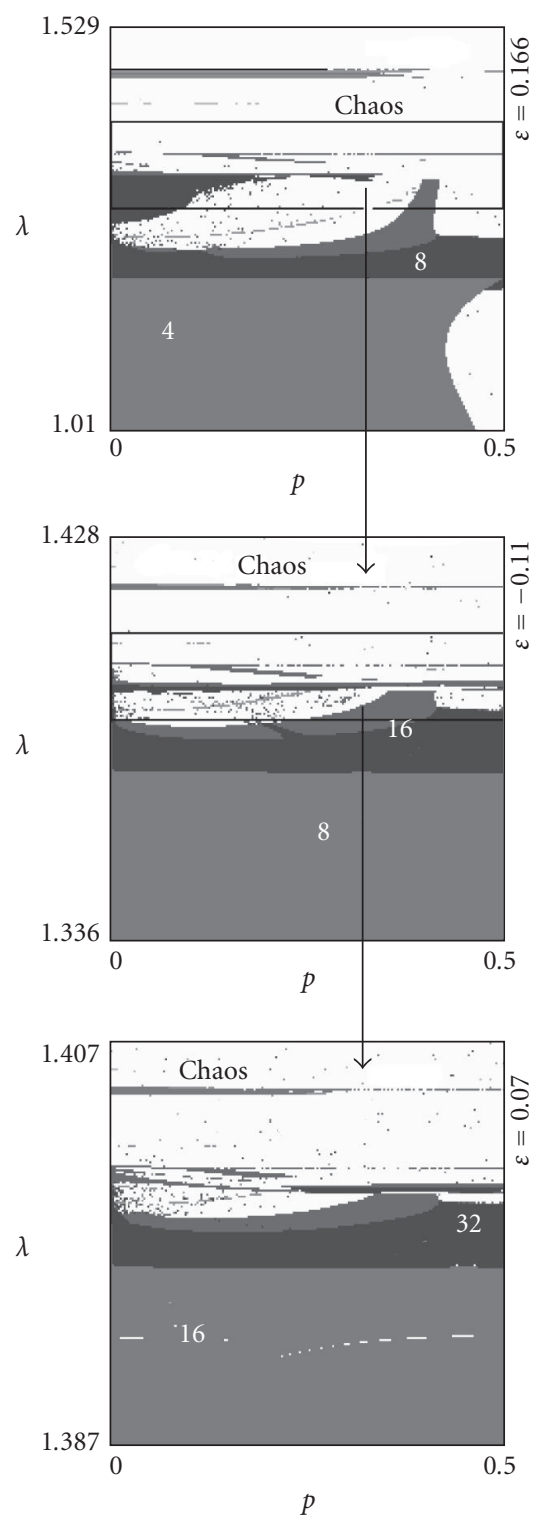

(b)

Figure 3.2. Illustrations of a scaling in the parameter space $\left(\lambda, p_{1}\right)$ for pure dissipative (left side) and pure inertial (right side) couplings for the following values of rest parameters $A=1.1249, B=0.375$, which correspond to bicritical point and $\varepsilon=0.25$ at initial time. The factor of scaling for $p_{1}$ is equal 1 .

section (see also the figures' captions), the factor of scaling for relative population numbers equals 1 . One can see that after the rescaling, similar structure in parameter space is observed. 
3.2. Dynamics of globally coupled maps. Let us turn now to consideration of dynamics of globally coupled maps with a pacemaker without preliminary assumptions about the cluster structure.

For characterization of regimes that have arisen in the course of dynamical evolution of the model of the globally coupled network that is dependent on parameters, it is appropriate to use a concept of phases introduced by Kaneko $[4,6,15,17]$. For a given point in the parameter space $\left(\lambda, A, B, \varepsilon_{1}, \varepsilon_{2}\right)$ we consider an ensemble of identical, mutually independent, globally coupled networks with random initial conditions. After a sufficiently large number of iterations, we analyse statistics of the number of clusters and determine the Kaneko phases as follows.

(i) Coherent phase: states with a single cluster (type-1 attractors) dominate in the ensemble.

(ii) Ordered phase: states with a few numbers of clusters (attractors of type 2) occur with larger probability.

(iii) Partially ordered phase: many-cluster attractors and few-cluster attractors appear with comparable probability.

(iv) Turbulent state: all observed attractors have a number of clusters of order $N$.

In Figure 3.3 the coherent phase is indicated by letter $\mathrm{C}$, ordered by letter $\mathrm{O}$, partially ordered by PO, and turbulent by letter T. The partially ordered phase, which corresponds to coexistence clusters with few and many elements, takes place near the boundaries of transition from one phase to another, for example, from coherent to ordered phase, or from ordered to turbulent. It occupies usually a very narrow domain in the parameter space.

One can observe that the largest part of the parameter space is occupied by a domain of coherent phase. In this domain of the coherent state the dynamics is described simply by (2.1) relating to two maps (master and slave) with unidirectional coupling. It means that presence of the pacemaker promotes synchronization in the network in a more wide region of the parameter space than that without pacemaker. In Figure 3.4 one can see the phase diagram for pure dissipative coupling (right) and pure inertial one (left) for case without pacemaker. All detections are similar for Figure 3.3. If coupling parameter $B=0$, the dynamics of (3.1) is really similar to case of network without pacemaker [4]. Also, we observe decrease of the domain of ordered phase due to presence of the pacemaker.

For small value of parameter of coupling and small $\lambda$, we observe the coherent phase. If $\varepsilon \approx 0$ and $\lambda=0.75$, then ordered phase has arisen. If $\lambda$ increases, the turbulent phase takes place. The main difference between two types of coupling is as follows. In dissipative coupling case for "big" value of $\varepsilon$ one can see only coherent phase, but for inertial one it may be also ordered or turbulent phase and ordered phase arises for $\lambda<\lambda_{c}$.

From scaling regularities stated for a model with two coupled slave systems driven by a pacemaker (Section 2), it follows that analogous regularities have to be intrinsic to the network of coupled slave elements because the global coupling may be regarded as the result of coupling of all possible pairs of elements. The scaling property may be formulated as follows. 


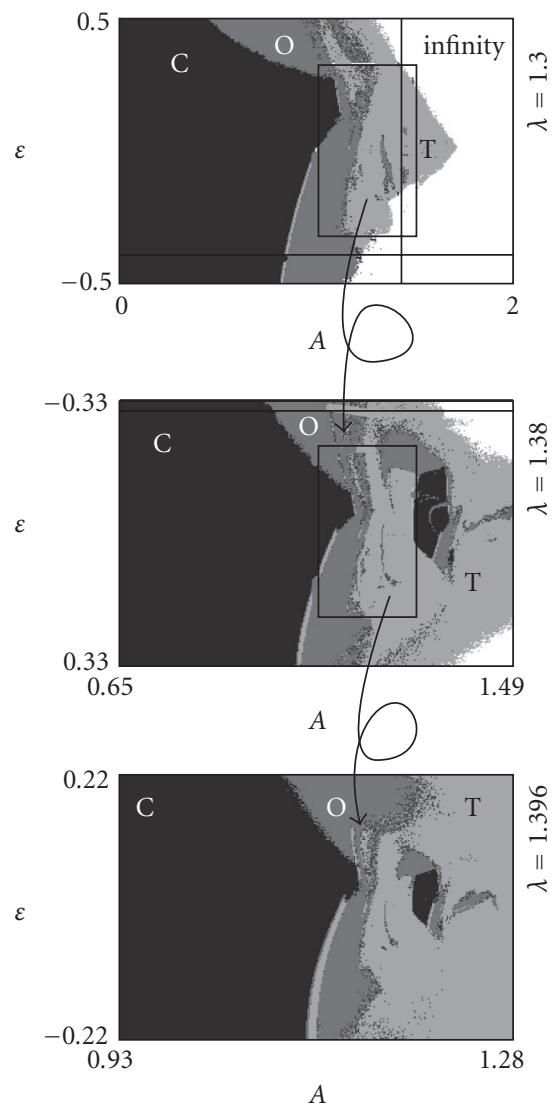

(a)

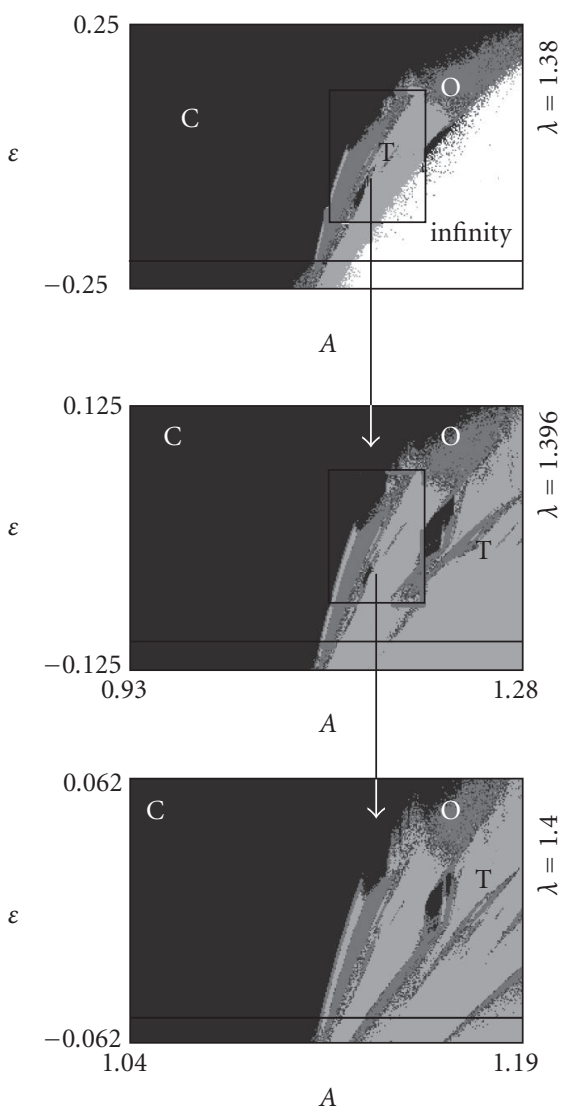

(b)

Figure 3.3. Illustrations of scaling in parameter space $(A, \varepsilon)$ for globally coupled maps with pacemaker for pure dissipative (left side) and pure inertial (right side) couplings. Letter $\mathrm{C}$ means coherent phase, letter $\mathrm{O}$ means ordered, and letter $\mathrm{T}$ - turbulent phase.

Let us suppose that at some parameter values $\left(\lambda, A, \varepsilon_{1}=C_{1}, \varepsilon_{2}=C_{2}-0.11855 C_{1}\right)$ near the bicritical point $\left(\lambda_{c}, A_{c}\right)$ we detect some Kaneko phase for ensemble of networks with random initial conditions from intervals $|x|<C,|y|<D$. Then, for an ensemble with random initial conditions in the intervals $|x|<C /|\alpha|,|y|<D /|\beta|$ at the point of parameter space $\left(\lambda_{c}+\left(\lambda-\lambda_{c}\right) / \delta_{1}, A_{c}+\left(A-A_{c}\right) / \delta_{2}, C_{1} / \beta, C_{2} / 2\right)$, we will observe the phase of the same kind, but with doubled time scale of the dynamics. Here $\alpha=2.5029$ and $\delta_{1}=4.6692$ are Feigenbaum's universal constants, and $\beta=-1.5053$ and $\delta_{2}=2.3927$ are universal constants associated with the bicritical behaviour.

In Figure 3.3 we illustrate this scaling property of the parameter space of the network at fixed master-slave coupling parameter $B=0.375$. As one can see in Figure 3.3, the scaling property is observed with good accuracy. The structure of parameter space after rescaling 


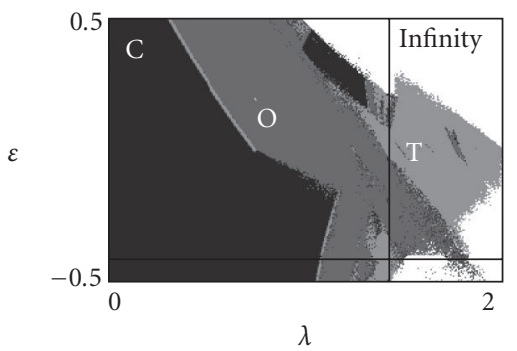

(a)

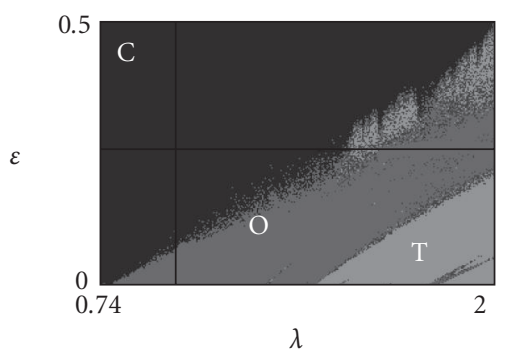

(b)

Figure 3.4. Phase diagrams for globally coupled maps without pacemaker for pure dissipative (a) and pure inertial (b) couplings. Letter $\mathrm{C}$ means coherent phase, letter $\mathrm{O}$ means ordered, and letter T-turbulent phase.

is not exactly similar to initial one, but the most important features, such as the lines of transitions to another phase, the "coherent windows" in turbulent phase, are reproduced.

\section{Conclusion}

In this paper we have introduced a model network of logistic maps with a pacemaker.

In Section 2 the renormalization group (RG) analysis for two coupled systems with a pacemaker have been developed. We conclude that in a general case the coupling is expressed via two basic types of coupling, dissipative and inertial.

For globally coupled system we also must investigate only two types of global coupling. We argued that clusterization phenomena and the Kaneko classification of phases suggested for the network with dissipative coupling [6] remain in our model.

In Sections 2 and 3 the two-cluster states were investigated in detail. We discover that dynamics of nonsymmetrically distributed two-cluster state is more complicated than the symmetrically distributed one. Variation of the parameter $p$ leads to a more developed chaos and the appearance of a complicated structure in the parameter plane (see Figure 3.1).

For globally coupled systems, the presence of a pacemaker leads to an increase in the size of the domain of coherent phase in the parameter space, that is, to more regular behaviour of network. In contrast to dissipative coupling, inertial coupling ensures more reach dynamics (see Figure 3.2, left—for pure dissipative global coupling, right—for pure inertial one).

We analyse a special type of critical behaviour near transition to chaos, the bicriticality, at the point of simultaneous transition to chaos in both subsystems. The illustrations of scaling for two coupled systems with a pacemaker are presented in this paper.

We have demonstrated scaling properties in the globally coupled system with a pacemaker. It follows from RG arguments developed previously for the system of two unidirectionally coupled maps that the scaling properties appear as an attribute of the universality class. This means that analogous dynamical regimes and the same scaling properties 
will be intrinsic not only to our logistic map model with a pacemaker but for any globally coupled network composed of period-doubling elements that relate to the bicritical class of universality. Thus, the results are expected to be common for globally coupled networks with a pacemaker of a different physical nature such as those in electronics, biology, economics, and so forth.

\section{Acknowledgments}

This work is partially supported by grant of RFBR 03-02-16074 and grant of BRHE Appendix-06-02.

\section{References}

[1] P. Cvitanović (ed.), Universality in Chaos, 2nd ed., Adam Hilger, Bristol, 1989.

[2] M. J. Feigenbaum, Quantitative universality for a class of nonlinear transformations, Journal of Statistical Physics 19 (1978), no. 1, 25-52.

[3] J. Garcma-Ojalvo, J. Casademont, and M. C. Torrent, Coherence and syncronization in diode-laser arrays with delayed global coupling, International Journal of Bifurcation and Chaos 9 (1999), no. 11, 2225-2229.

[4] A. S. Ivanova and S. P. Kuznetsov, Scaling at the onset of chaos in a network of logistic maps with two types of global coupling, Nonlinear Phenomena in Complex Systems 5 (2002), no. 2, 151-154.

[5] K. Kaneko, Chaotic but regular posi-nega switch among coded attractors by cluster-size variation, Physical Review Letters 63 (1989), no. 3, 219-223.

[6] Clustering, coding, switching, hierarchical ordering, and control in a network of chaotic elements, Physica D 41 (1990), no. 2, 137-172.

[7] __ Relevance of dynamic clustering to biological networks, Physica D 75 (1994), no. 1-3, $55-73$.

[8] S.-Y. Kim, Bicritical behavior of period doublings in unidirectionally coupled maps, Physical Review E 59 (1999), no. 6, 6585-6592.

[9] S.-Y. Kim and H. Kook, Renormalization analysis of two coupled maps, Physics Letters A 178 (1993), no. 3-4, 258-264.

[10] S.-Y. Kim and W. Lim, Bicritical scaling behavior in unidirectionally coupled oscillators, Physical Review E 63 (2001), no. 3, 036223.

[11] I. Z. Kiss, W. Wang, and J. L. Hudson, Population of coupled electrochemical oscillators, Chaos 12 (2002), no. 1, 252-263.

[12] S. Yu. Kourtchatov, V. V. Likhanskii, A. P. Napartovich, F. T. Arecchi, and A. Lapucci, Theory of phase locking of globally coupled laser arrays, Physical Review A 52 (1995), no. 5, 4089-4094.

[13] S. P. Kuznetsov, Renormalization group, universality and scaling in dynamics of coupled map lattices, Theory and Applications of Coupled Map Lattices (K. Kaneko, ed.), John Wiley \& Sons, Chichester, 1993, pp. 51-94.

[14] A. P. Kuznetsov, S. P. Kuznetsov, and I. R. Sataev, Bicritical dynamics of period-doubling systems with unidirectional coupling, International Journal of Bifurcation and Chaos 1 (1991), no. 4, 839-848.

[15] S. C. Manrubia and A. S. Michailov, Very long transients in globally coupled maps, Europhysics Letters 50 (2000), no. 5, 580-586.

[16] N. Oketani and T. Ushio, Chaotic synchronization of globally coupled maps with an application in communication, International Journal of Bifurcation and Chaos 6 (1996), no. 11, 2145-2152.

[17] O. Popovych, A. Pikovsky, and Yu. Maistrenko, Cluster-splitting bifurcation in a system of coupled maps, Physica D 168/169 (2002), 106-125. 
[18] R. F. Schmidt and G. Thews, Human Physiology, Springer, New York, 1983.

[19] V. K. Vanag, L. Yang, M. Dolnik, A. Zhabotinsky, and I. R. Epstein, Oscillatory cluster patterns in a homogeneous chemical system with global feedback, Nature 406 (2000), no. 6794, 389-391.

Anna S. Ivanova: Department of Nonlinear Processes, Saratov State University, Astrakhanskaya 83, Saratov 410026, Russia

E-mail address: anna-ivanova@yandex.ru

Sergey P. Kuznetsov: Laboratory of Theoretical Nonlinear Dynamics, Saratov Branch of Institute of Radio-Engineering and Electronics, Russian Academy of Sciences, Zelenaya 38,

Saratov 410019, Russia

E-mail address: kuznetsov@sgu.ru

Andrew H. Osbaldestin: Department of Mathematics, University of Portsmouth, Portsmouth PO1 3HE, UK

E-mail address: andrew.osbaldestin@port.ac.uk 


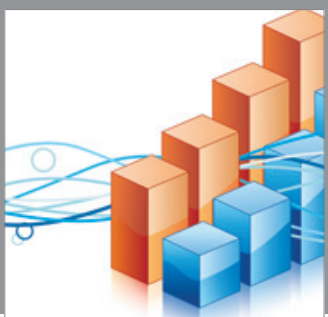

Advances in

Operations Research

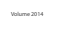

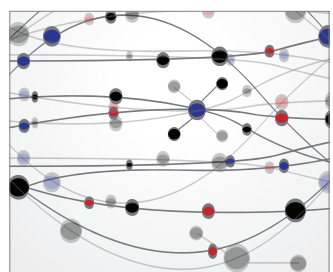

\section{The Scientific} World Journal
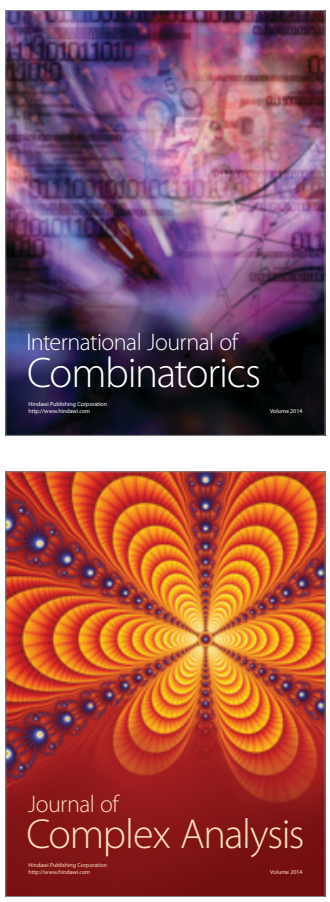

International Journal of

Mathematics and

Mathematical

Sciences
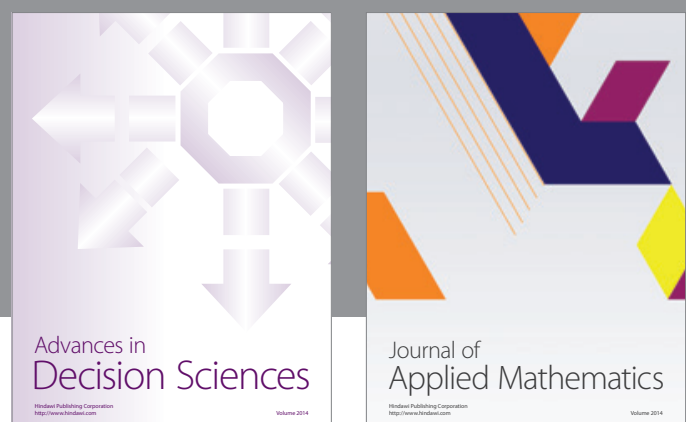

Journal of

Applied Mathematics
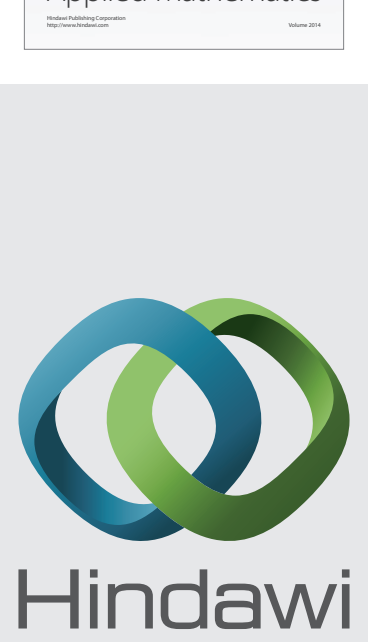

Submit your manuscripts at http://www.hindawi.com
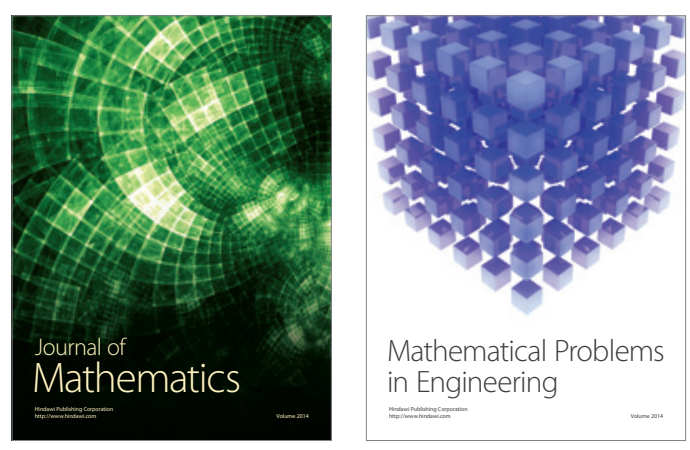

Mathematical Problems in Engineering
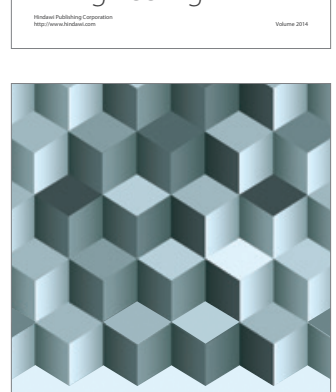

Journal of

Function Spaces
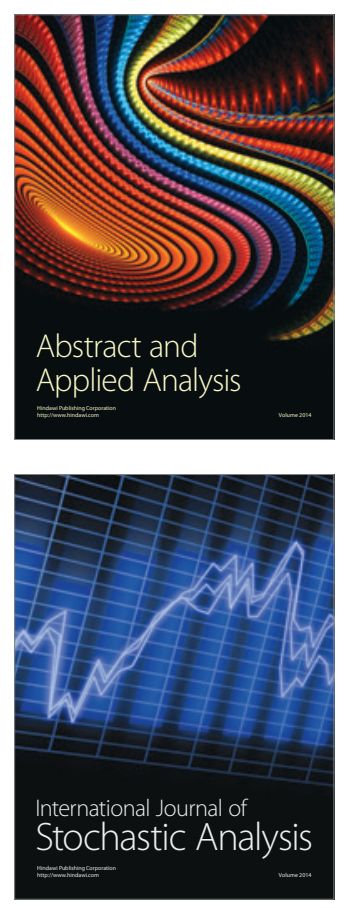

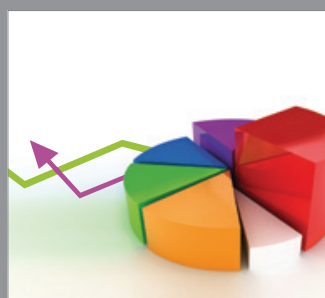

ournal of

Probability and Statistics

Promensencen
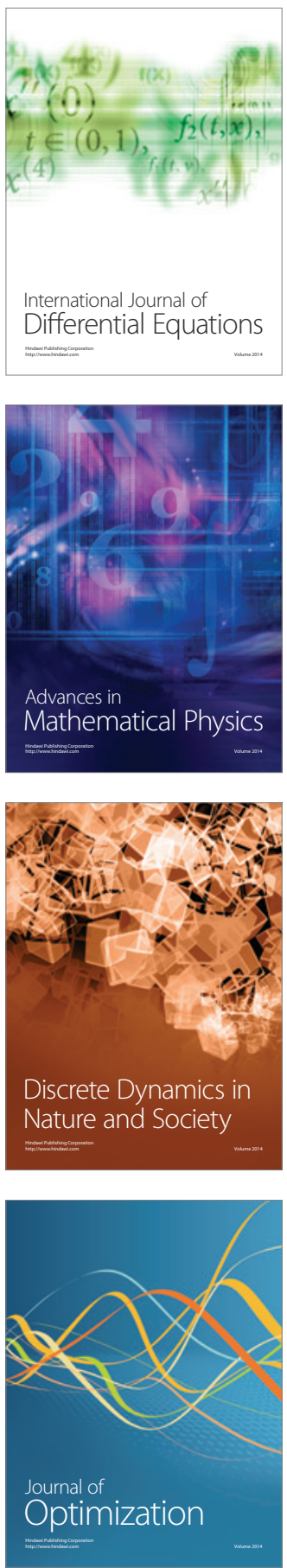